\title{
Mediastinal schwannoma: A case report
}

Shrestha DK' ${ }^{1}$, Singh DR ${ }^{2}$

${ }^{1}$ Resident, ${ }^{2}$ Associate Professor, Department of Surgery, Kathmandu Medical College Teaching Hospital, Kathmandu, Nepal

\section{Abstract}

Schwannomas are neurogenic tumours arising from Schwann cells of neural sheath. The peak incidence of these tumours is in 30 s to 50 s of life, with men and women being equally affected. Schwannoma is an encapsulated tumour which distinguishes it from neurofibroma without encapsulation. With both Schwannoma and neurofibroma, surgical excision results in cure. Our patient, twenty year male, presented with mild, dull aching pain over right antero-lateral chest and non-productive cough associated with breathlessness on physical exertion. Computed tomography of the chest was suggestive of neurogenic tumour (differential diagnoses: ganglioneuroma/ganglioneuroblastoma). The mass was excised via open right posterolateral thoracotomy and was sent for histopathological examination after which he was diagnosed as having Mediastinal Schwannoma. Mediastinal schwannomas, although generally benign and asymptomatic, should be excised upon discovery to prevent the development of life-threatening complications.

Key words: Benign, Neurogenic tumours, Schwannoma, Surgical excision

\section{INTRODUCTION}

chwannomas are neurogenic tumours arising $\checkmark$ from Schwann cells of neural sheath. Mediastinal schwannomas most frequently arise in a paravertebral location from sympathetic trunks or intercostal nerves ${ }^{1}$. Fourty five percent of schwannomas occur in the head and neck, with nine per cent occurring in the mediastinum ${ }^{2}$. They are benign, slow growing neoplasms that frequently arise from a spinal nerve root, but can involve any thoracic nerve. They arise from the nerve sheath and extrinsically compress the nerve fibres. The peak incidence of these tumours is in 30 s to 50 s of life, with men and women being equally affected $^{3}$. Schwannoma is firm, well encapsulated and generally benign tumour which distinguishes it from Neurofibroma without encapsulation. Two characteristic histologic components of benign Schwannoma exist and are referred as Antoni type $A$ and Antoni type $B$ regions. Antoni type A regions contain compact spindle cells with twisted nuclei and nuclear palisading. Antony type B regions contain loose and myxoid connective tissue with haphazard cellular arrangement. These characteristics allow them to be distinguished from malignant, fibrosarcomatous tumours that have no Antoni feature.

\section{Address for correspondence}

Dr. Dipendra Kumar Shrestha

Resident, Department of Surgery

Kathmandu Medical College Teaching Hospital,

Sinamangal, Kathmandu

E-mail: dipen_sk@hotmail.com
Our patient presented with mild, dull aching, right antero-lateral chest pain and non-productive cough associated with shortness of breath on physical exertion with no any other features of complication. He was diagnosed to have postero-superior mediastinal mass by contrast enhanced computerized tomography (CECT) scan chest and found to be Mediastinal Schwannoma on histopathological examination. He underwent open right posterolateral thoracotomy, accessed via incision over right fourth intercostal space under general anaesthesia.

\section{CASE REPORT}

A twenty year male from Argakhanchi presented with mild, dull aching pain over right antero-lateral chest and non-productive cough associated with breathlessness on physical exertion. At presentation his general condition was normal and his vital parameters were stable. Systemic examination revealed no abnormalities. There was no localized swelling, deformity, tenderness, local rise in temperature and skin over the right chest wall was normal. Routine blood investigation showed Haemoglobin: $9.1 \mathrm{gm} / \mathrm{dL}$, Total count: $4000 / \mathrm{mm}^{3}$, Platelet count: $126,000 / \mathrm{mm}^{3}$, Differential count with neutrophil $70 \%$, lymphocyte $28 \%$ and Eosinophil 1\%, Random blood sugar: $89 \mathrm{mg} / \mathrm{dL}$, Blood Urea: $24 \mathrm{mg} / \mathrm{dL}$, Serum creatinine: $0.6 \mathrm{mg} / \mathrm{dL}$, Sodium: $144 \mathrm{mEq} / \mathrm{L}$ and Potassium: $3.3 \mathrm{mEq} / \mathrm{L}$. Chest X-ray showed round hyperdense shadow over the right apical region in postero-anterior view and over the posterior region in lateral view (Figure 
1). Ultrasonography (USG) chest showed oval tissular pattern on lung parenchyma at the apical region (Figure 3). He had already undergone Contrast enhanced Computerised tomography (CECT) scan (Figure 2) chest which showed $11 \times 10 \times 9 \mathrm{~cm}^{3}$ mildly enhancing sharply marginated mediastinal mass along the right costal, paravertebral and paratracheal region with superior extension showing extrinsic mass effect and apparent extrathoracic extension suggestive of neurogenic tumour (differential diagnoses: ganglioneuroma/ ganglioneuroblastoma). Pulmonary Function test (PFT) revealed fixed upper airway obstruction with the ratio of Forced expiratory volume in one second (FEV1) to Forced vital capacity (FVC) $65.3 \%$ (less than 70\%), reduced FEV1 $55.5 \%$ (less than 80\%), normal FVC 96\% (more than $80 \%)$, normal Vital capacity (VC) and flow volume loop showing adequate volume but severe reduction in flow on exhalation with characteristic flattening with wellpreserved inspiratory limb. Patient was diagnosed to have mass over right postero-superior mediastinum and was planned for excision of the mass. He underwent open right posterolateral thoracotomy, via incision over right fourth intercostal space under general anaesthesia. Grossly round, smooth, rubbery mass (Figure 4) of about $13 \times 11 \times 10 \mathrm{~cm}^{3}$ was seen over the postero-superior aspect of the right lung with avulsion of the lung around the mass and depression of the mass over the right lung. Surrounding structures like Aorta, Inferior venacava and trachea were not involved. Histologically, mass was consistent with Schwannoma characterised by encapsulated variegated appearance exhibiting cellular spindle cells with fascicular and storiform patterns interrupted by hypocellular oedematous and hyalinised areas with focal degenerative nuclear atypia. During the procedure and following procedure patient status was normal. Patient was stable and shifted to Intensive Care Unit for monitoring as resection of huge thoracic tumours may result in arrhythmia and post-operative bleeding. He was discharged on ninth post-operative day and had a steady recovery.
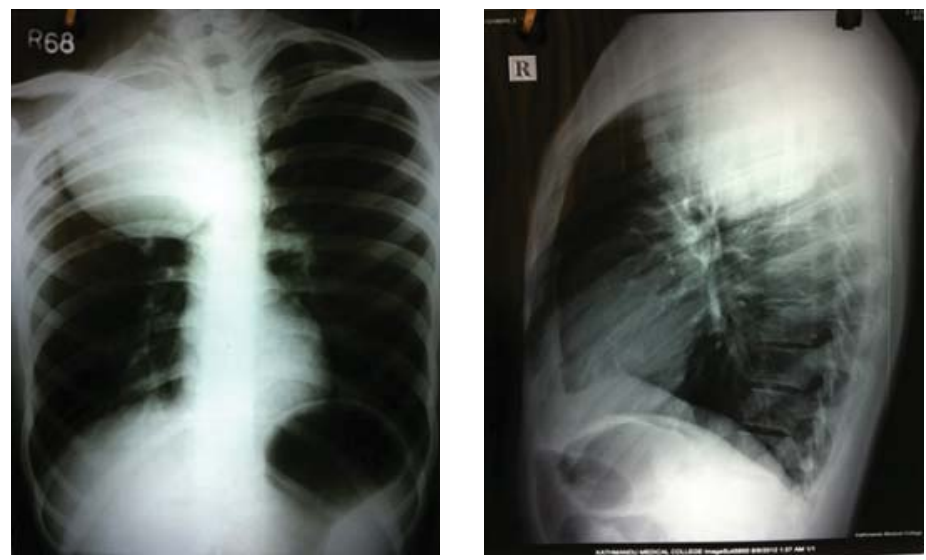

Figure 1: Round hyperdense shadow in right Apical region in Posteroanterior view and in posterior region in Lateral chest X-ray

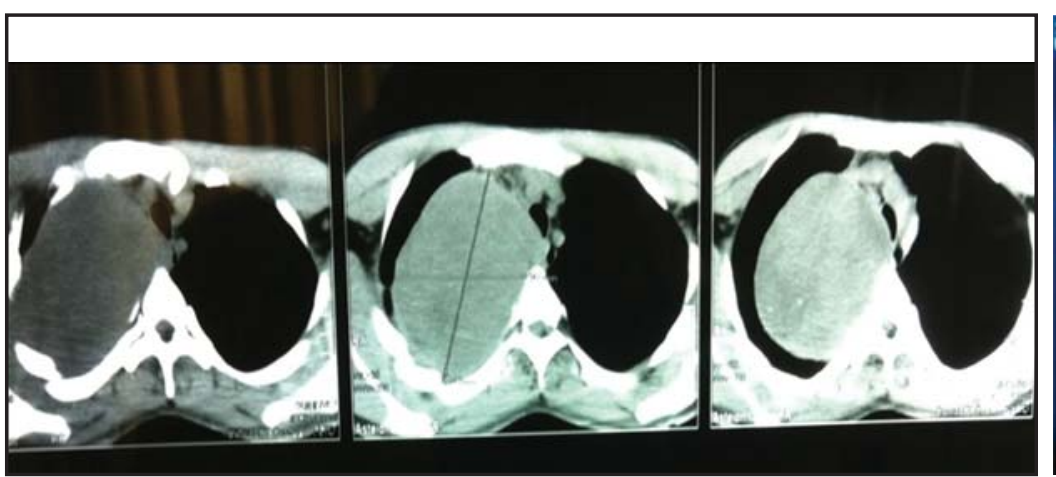

Figure 2: CECT chest showing mildly enhancing sharply marginated mediastinal mass

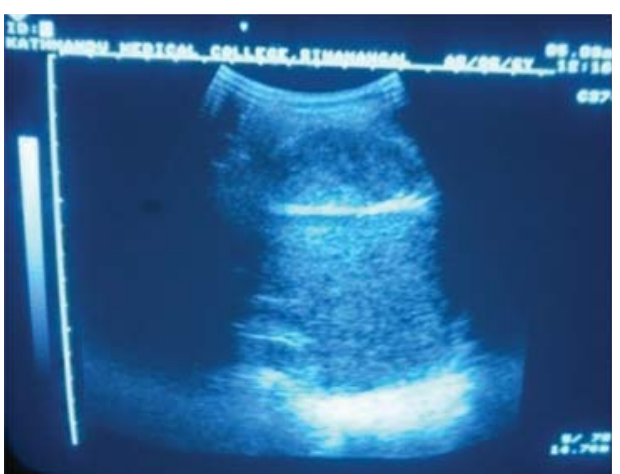

Figure 3: USG chest: Oval Tissular pattern 


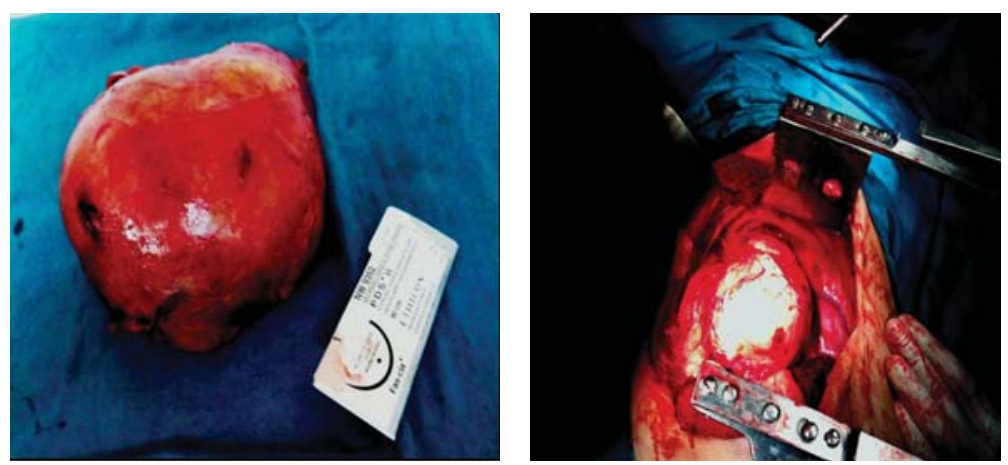

Figure 4: Round, smooth and rubbery mediastinal mass

\section{DISCUSSION}

Benign neurogenic tumours are mostly located in the posterior mediastinum. These include nerve root tumours (Schwannomas or neurofibromas), sympathetic ganglion tumours (neuroblastomas, ganglioneuroblastomas, ganglioneuromas) and paragangliomas (chemodectomas and pheochromocytomas). Among these tumours benign Schwannomas are the most frequently encountered type in clinical practice.

The peak incidence of these tumours is in 30 s to 50 s of life, with men and women being equally affected. In children and infants, neurogenic tumours are the most commonly occurring tumour. Approximately two thirds of mediastinal masses are symptomatic in the paediatric population, while only approximately one third produce symptoms in adults. When considering all age groups, nearly $55 \%$ of patients with benign mediastinal masses are asymptomatic at presentation, compared to only approximately $15 \%$ of those in whom masses are found to be malignant ${ }^{4}$. Rarely patient with left posterior Mediastinal Schwannoma may present with cardiac tamponade, a life threatening condition ${ }^{5}$.

Radiological tools like CT and magnetic resonance imaging of the chest and spine are useful in determining the exact anatomic location of the mass, as well as exclude any vascular origin, local and intraspinal invasion of the mass. These modalities are also very useful in excluding other differential diagnoses of posterior mediastinal mass.
Mediastinal Schwannomas most frequently arise in a paravertebral location from sympathethic trunks or intercostal nerves ${ }^{6}$. Radical surgical excision of the mass by thoracoscopy or thoracotomy is the treatment of choice ${ }^{7}$. However, there is no way to determine the malignancy of the lesion; even benign tumours can grow to large dimensions and cause compression symptoms ${ }^{8}$. The tumour will continue to increase in size if left untreated; hence, prompt management would affect the prognosis. Thoracotomy via the posterolateral approach has been in practice for long as a conventional surgical technique for resection of these posterior mediastinal masses ${ }^{9}$. Now days, video-assisted thoracoscopic surgery is the preferred technique for the diagnosis and management of benign posterior mediastinal masses, as it is less invasive and results in fewer lung complications and a shorter hospital stay. However, malignant lesions are best approached via open thoracotomy ${ }^{10}$. Patients with benign neurogenic tumours have excellent survival prospects following complete resection, whereas those with malignant tumours have a poorer prognosis ${ }^{11}$.

\section{CONCLUSION}

Schwannoma is a benign and slowly growing neurogenic tumour of mediastinum. Though mostly diagnosed incidentally but sometimes patient present with chest pain, cough and shortness of breath and rarely with cardiac tamponade, a life threatening complication of mediastinal masses like Schwannoma; timely intervention by open thoracotomy or thoracoscopy can lead to cure of these benign mediastinal pathology. 


\section{REFERENCES}

1. Marchevsky AM. Mediastinal tumors of peripheral nervous system origin. Semin Diagn Pathol. 1999;16(1):65-78.

2. Dasgupta TK, Brasfield RD: Benign solitary Schwannomas (neurilemomas). Cancer. 1969;24(2):355-66.

3. Varghese TK, Lau CL. The mediastinum. In:Townsend $\mathrm{CM}$, Beauchamp RD, Evers BM, Mattox KL, editors. Sabiston Textbook of Surgery. 18th ed. Vol 2. Philadelphia: Saunders Elsevier; c2008. p.1684-5.

4. Cohen LM, Schwartz AM, Rockoff SD. Benign schwannomas: pathologic basis for CT in homogeneities. Am J Roentgenol. 1986;147:141-3.

5. Kato M, Shiota S, Shiga K, Takagi H, Mori H, Sekiya $M$, et al. Benign giant mediastinal schwannoma presenting as cardiac tamponade in a woman: a case report. J Med Case Rep. 2011 Feb 14;5:61.
6. Strollo DC, Rosado-de-Christensonml, Jett JR. Primary mediastinal tumors: part II. Tumors of the middle and posterior mediastinum. Chest. 1997;112(5):13441357. doi: $10.1378 /$ chest.112.5.1344

7. Fierro N, D'ermo G, Di Cola G, Gallinaro LS, Galassi G. Asian Cardiovasc Thorac Ann. 2003;11:72-3.

8. Marchevsky AM. Mediastinal tumors of peripheral nervous system origin. Semin Diagn Pathol. 1999;16(1):65-78.

9. Topçu S, Alper A, Gülhan E, et al. Neurogenic tumours of the mediastinum: a report of 60 cases. Can Respir J. 2000;7:261-5.

10. Reardon MJ, Conklin LD, Fabre J, Reardon PR, Letsou GV. Thoracoscopic approach to posterior mediastinal neurogenic tumors in the adult. J Laparoendosc Adv Surg Tech. 1999;9:187-92.

11. Reeder LB. Neurogenic tumors of the mediastinum. Semin Thorac Cardiovasc Surg. 2000;12:261-7. 